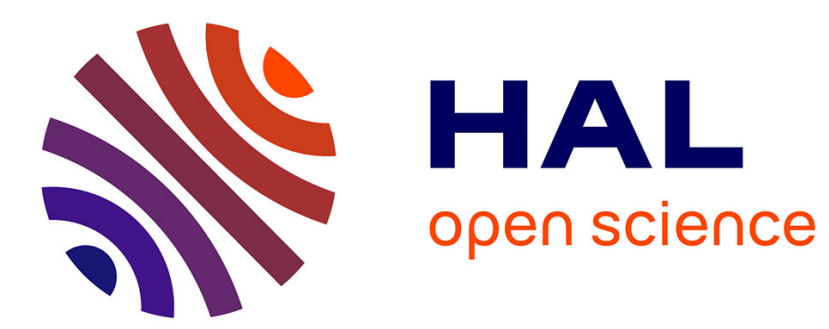

\title{
Thèses en France et science ouverte
}

Maité Roux

\section{To cite this version:}

Maïté Roux. Thèses en France et science ouverte. Arabesques, 2019, 93, pp.10. 10.35562/arabesques.553. hal-03225443

HAL Id: hal-03225443

https://hal.science/hal-03225443

Submitted on 12 May 2021

HAL is a multi-disciplinary open access archive for the deposit and dissemination of scientific research documents, whether they are published or not. The documents may come from teaching and research institutions in France or abroad, or from public or private research centers.
L'archive ouverte pluridisciplinaire HAL, est destinée au dépôt et à la diffusion de documents scientifiques de niveau recherche, publiés ou non, émanant des établissements d'enseignement et de recherche français ou étrangers, des laboratoires publics ou privés. 


\section{Thèses en France et science ouverte}

\section{Documents à la nature juridique particulière, les thèses pourraient être à l'avant-poste de la diffusion en science ouverte.}

Les thèses de doctorat françaises, tout à la fois œuvres de l'esprit, données de la recherche et documents administratifs, bénéficient depuis le $\mathrm{XIX}$ siècle d'un régime réglementaire favorable à leur ouverture. Assurée tout d'abord par l'envoi de reproductions imprimées, puis microfichées ${ }^{1}$, la diffusion des thèses a été rendue obligatoire au sein de la communauté universitaire dès $1841^{2}$.

L'essor d'internet et des supports électroniques dans les années 2000 a permis d'élargir le périmètre de diffusion à l'ensemble de la communauté nationale et internationale ${ }^{3}$, mais en soumettant cette ouverture à l'accord des auteurs. Aujourd'hui coexistent donc : un corpus comprenant l'ensemble des thèses électroniques, mais dont l'accès est conditionné à l'appartenance à une communauté d'utilisateurs définie, et un corpus librement accessible en ligne, mais incomplet.

\section{NUANCES D'OUVERTURE EN OPEN ACCESS}

$73 \%$ des quelques 85000 thèses de doctorat nativement numériques ${ }^{4}$ sont disponibles en accès ouvert sur internet. Le pourcentage annuel de thèses en accès restreint baisse de manière continue depuis 2013 (29\% pour les thèses soutenues en 2013 , contre $23 \%$ pour les thèses soutenues en 2017), ce qui indique que la pratique de l'open access progresse chez les jeunes chercheurs. En revanche, on observe que les restrictions temporelles (embargo ou confidentialité), qui viennent retarder la diffusion, connaissent une évolution inverse. Si l'on considère ainsi les thèses soutenues en 2013, $16 \%$ des cas de diffusion sur internet étaient assortis d'une restriction temporelle, contre $20 \%$ pour les thèses soutenues en 2017. La tendance est à ouvrir plus, mais plus tard.

Il faut aussi noter que la sensibilité à l'open access diffère beaucoup d'une discipline à l'autre : $84 \%$ des thèses en sciences fondamentales et appliquées sont disponibles en libre accès, contre $49 \%$ seulement en lettres et sciences humaines. Au sein même de ces grands ensembles, les disparités sont fortes. Le taux de thèses disponibles en accès ouvert est très élevé en informatique et mathématiques (92\%) ou en physique (91\%), alors qu'il tombe à $77 \%$ pour la biologie et à $70 \%$ pour les sciences médicales.
Du côté des humanités, les thèses sont davantage diffusées en géographie (69\%), sciences de l'éducation $(67 \%)$ et économie (66\%), qu'en droit (39\%), arts $(38 \%)$, lettres et histoire (37\%). Inversement, la pratique de l'embargo et de la confidentialité est plus répandue en sciences exactes (19\% en moyenne) qu'en sciences humaines

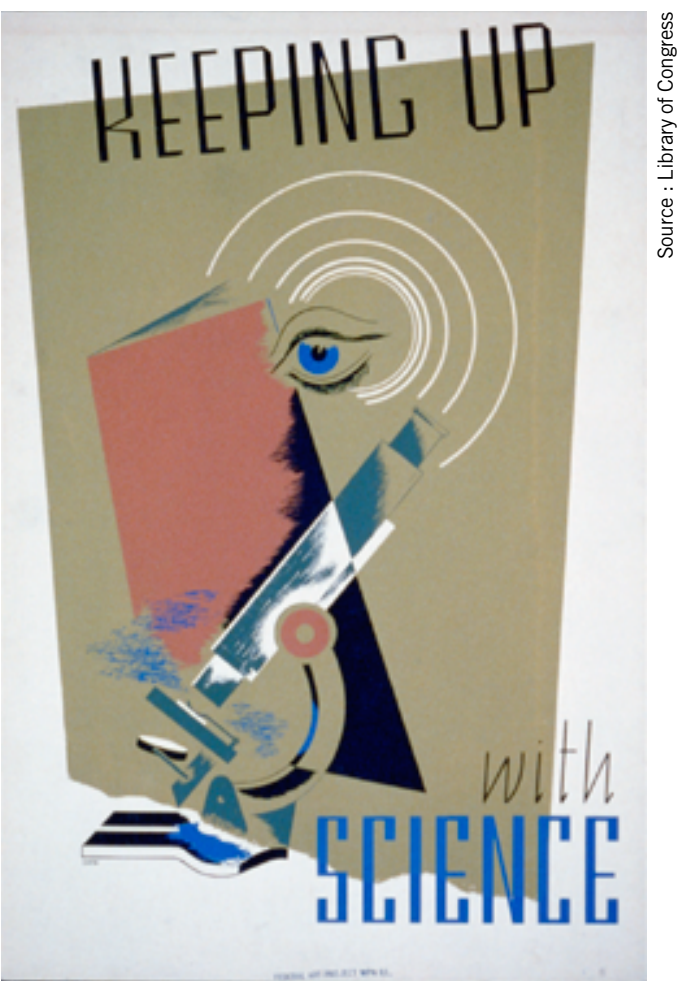

(9\%). Dans le premier cas, on retarde la diffusion pour répondre à l'injonction de publication ou protéger temporairement des données, dans le second, le refus de diffuser est plus définitif.

\section{LEVER LES OBSTACLES AU LIBRE ACCÈS}

Il est essentiel de sensibiliser les docteurs aux avantages de l'open access (valorisation de leurs travaux, protection renforcée contre le plagiat du fait d'une plus grande visibilité) et de lever leurs réticences par des solutions de contournement. Le docteur et ses partenaires souhaitent déposer un brevet, publier les résultats obtenus dans une revue ou voir la thèse commercialisée par un éditeur : on proposera une mise sous embargo. Le corps du texte ou les annexes de la thèse contiennent des éléments non diffusables pour des raisons de droits (illustrations, extraits d'œuvres musicales ou audiovisuelles) ou de respect de la vie privée (entretiens) : il suffira d'anonymiser les données personnelles et d'expurger les éléments problématiques. Diffuser plus tard, diffuser partiellement, l'important est d'ouvrir les données autant que faire se peut.

Aujourd'hui, alors que le Plan national pour la science ouverte envisage d'élargir aux thèses les mesures prévues pour l'ouverture des publications, il ne semble pas irréaliste de viser une libération à $90 \%$ du corpus, ce qui placerait ce type de documents aux avant-postes de la science ouverte.

\section{MAÏTÉ Roux \\ Chef du service des thèses Abes m.roux@abes.fr} Keeping up with science,
1936

[1] Arrêté du 25 septembre 1985.

[2] Arrêté du 7 décembre 1841.

[3] Arrêté du 7 août 2006 puis arrêté du 25 mai 2016

[4] II s'agit des thèses archivées dans le cadre du circuit de dépôt national STAR. Les copies électroniques de thèses imprimées ne sont pas comptabilisées. Theses.fr en dénombre 33500 , soit $10 \%$ des thèses archivées au format papier. 